\title{
Reduced Order Models of Integrated RF Spiral Inductors with Geometrical and Technological Automatic Parameterization
}

\author{
Abhishek Garg, Ranjit Gharpurey ${ }^{\dagger}$, Edoardo Charbon \\ Processor Architecture Laboratory, Ecole Polytechnique Federale de Lausanne, \\ 1015, Lausanne, Switzerland. \\ ${ }^{\dagger}$ Department of EECS, University of Michigan at Ann Arbor, MI 48109, USA. \\ abishek.garg, edoardo.charbon@epfl.ch,ranjitg@eecs.umich.edu
}

\begin{abstract}
In this paper a method is presented for automatically generating a parameterized model of integrated inductors accounting for geometry and substrate effects. A multiparameter Krylovsubspace based moment matching method is used to reduce the three-dimensional integral equations describing the EM behavior of the inductor over the substrate. Parameterization enables optimization of geometry and substrate technology simultaneously.
\end{abstract}

\section{INTRODUCTION}

Integrated radio-frequency $(\mathrm{RF})$ transceivers operating at frequencies of a few hundred megahertz up to a few tens of gigahertz often include one or more planar inductors. Accurate modeling of such components is key to quick design cycles and overall shorter time-to-market. Accurate inductor parameterization can be an effective tool to perform a number of optimizations at circuit and layout levels, thus ensuring higher overall robustness and better yields.

Analytical expressions fully characterizing spiral inductors of the type used in ICs are difficult to obtain. The reasons are in the complexity of the EM interactions between the various subcomponents of the inductor and in their nonlinear dependence from geometrical and electrical parameters. Therefore a full numerical solution of Maxwell's equations for the 3D inductor geometry is generally the method of choice. The main limitation of this approach, in addition to its computational complexity, is a lack of flexibility, since a full solution of the equation is required with even minimal variations of geometrical and/or technological parameters. However, design explorations, what-if analyses, and yield optimization often require hundreds or thousands of such parameter combinations.

An alternative to the fully numerical approach is the generation of parameterized reduced-order models. This approach has been proposed before for interconnect delay estimation [2],[3],[10] and RF parasitics [13]. More recently, automated methods for the generation of reduced order models targeting integrated spiral inductors have been proposed [1]. The core of the approach is Krylov-subspace based moment-matching method that reduces the integral equation describing the 3D EM behavior of the inductor. While it accounts for distributed capacitance, skin depth, and inductive cross-coupling, this method intentionally ${ }^{1}$ ignores substrate-induced parasitic effects, such as capacitive coupled noise, Eddy currents, etc. Other researchers have addressed these issues [9],[11]. 1 The reason for this choice was reportedly computational
tractability.
The technique proposed in [9] enables modeling the substrate effects, but the inability to construct a parameterized system of equations in [9] restricts its application to only small design space exploration. The complete simulation of RF inductor for small changes in physical dimensions makes it less attractive for large design space exploration.

In this paper, we propose a substrate-aware Krylov-subspace based moment matching method for inductor model parameterization. The method automatically performs standard multiparameter moment matching for the reduction of the integral equations describing the inductor. The substrate region underlying the inductor is accurately modeled using integral equation based Boundary Element Method (BEM). We provide a technique for merging the circuit resulting from substrate mesh with the Partial Element Equivalent Circuit (PEEC) of the inductor and show how the resulting circuit can be solved using existing techniques [1] for parameterization and model order reduction.

The paper is organized as follows. In section 2, we describe the model order reduction scheme for RF spiral inductors. In section 3 , a technique is given for computation of substrate parasitics. In section 4, we describe how we include substrate noise in the PEEC formulation for spiral inductors. Finally in section 5, we give the experimental results to support the suitability of our model.

\section{Modeling RF Inductors}

A spiral inductor can be represented as set of conductors connected to each other back-to-back to form a polygonal structure. Inside each conductor, the current density and charge concentration can be modeled by the following system [5,6] of integral equations:

$$
\begin{gathered}
\frac{J(r)}{\sigma}+j \omega \frac{\mu}{4 \pi} \int_{V} \frac{J\left(r^{\prime}\right)}{\left|r-r^{\prime}\right|} d r^{\prime}=-\nabla \phi, \\
\frac{1}{4 \pi \varepsilon} \int_{S} \frac{\rho\left(r^{\prime}\right)}{\left|r-r^{\prime}\right|} d r^{\prime}=\phi\left(r^{\prime}\right), \\
\nabla \cdot J(r)=0 \\
\hat{n} \cdot J(r)=j \omega \rho(r)
\end{gathered}
$$

In the above equations, $J$ is the unknown current distribution, $\rho$ is the unknown surface charge distribution. $\sigma$ is the conductivity of conductor and $\mu$ and $\varepsilon$ are the free space permeability and permittivity respectively. $\Phi$ is the potential function at point defined by vector ' $r$ ', $\omega$ is the angular frequency of operation. $V$ and $S$ are the Volume and surface area respectively. 
The first step towards solving this set of equations for $J$ and $\rho$ is the discretization. Since the length of conductors is large as compared to width and depth of the conductors, we can use the PEEC formulation to discretize the conductors. To model the current flows, the conductors are divided into filaments of rectangular cross-sections, such that the current inside each filament can be assumed to be parallel to the surface. To model the charge accumulation on the surface of conductors, the surface is divided into small 2D rectangular equi-potential panels. Dividing each conductor of the spiral inductor into filaments and panels, we obtain the circuit as shown in figure 1(a).

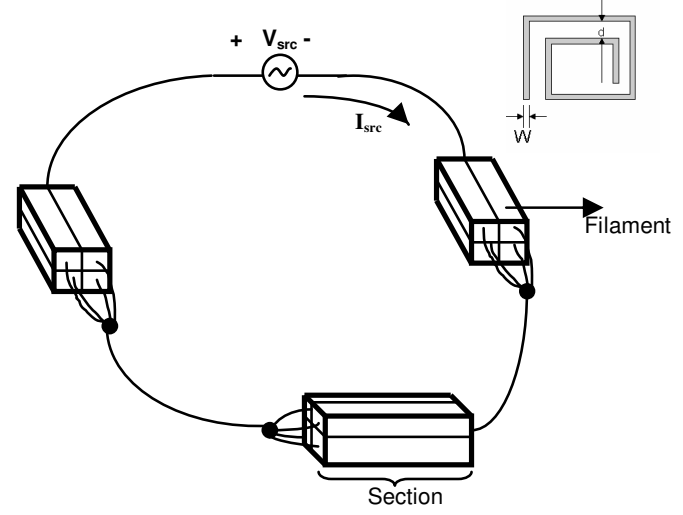

Fig 1(a) : Break up of spiral inductor into filaments



Fig 1(b) : PEEC circuit for Spiral Inductor

The resulting PEEC is shown in figure 1(b). It can be solved using the mesh based analysis given in [4]. The analysis, leads to the set of linear equations which can be represented in the matrix form as:

$$
\begin{gathered}
{\left[\begin{array}{cccc}
Z_{m f} & Z_{m f s} & 0 & 0 \\
Z_{m f s}^{T} & Z_{m s} & 0 & M_{p s} \\
0 & 0 & 0 & M_{p} \\
0 & -p M_{p s}^{T} & -p M_{p}^{T} & s I
\end{array}\right]\left[\begin{array}{c}
I_{m}^{f} \\
I_{m}^{s} \\
I_{m}^{p} \\
V_{b}^{p}
\end{array}\right]=\left[\begin{array}{c}
V_{s}^{f} \\
V_{s}^{s} \\
V_{s}^{p} \\
0
\end{array}\right]} \\
Z x=B u
\end{gathered}
$$

In equation (5), $Z(i, j)$ stands for the impedance in mesh $i$ due to current in mesh $j$.

In equation (6), $Z$ is the impedance matrix of the circuit, state vector $x$ represents the internal state of the circuit, $u$ is the voltage source vector and matrix $B$ is the incidence matrix of these voltage sources on different meshes in the network. Once we have the equations in the form of (6), a parameterization and model order reduction as described in $[1,2]$ is used to obtain the set of equations (7) and (8):

$$
\begin{gathered}
\left(s_{0} V^{T} Z_{0} V+s_{1} V^{T} Z_{1} V+\ldots \ldots .+s_{p} V^{T} Z_{p} V\right) \hat{x}=V^{T} B u \\
y=B^{T} V \hat{x}
\end{gathered}
$$

where, $V$ is the projection matrix of size $n \times m(\mathrm{~m}<<\mathrm{n})$ and $\hat{x}$ is the reduced state space. Vector $y$ gives the current flowing through the voltage source in the inductor. In (7), $s_{i}$ 's are the parameters used for parameterizing the system and $p$ is the number of parameters in the system. The projection matrix, $V$ is generated using the krylov subspace method, the details of which can be found in [2].

Once this reduced set of equations has been obtained, different configurations of inductors can be tried by changing the values of the parameters. Every time the parameters are changed, few simple scalar-matrix multiplications and matrix-matrix addition operations are required. Finally matrix inversion is performed in (7) to obtain the reduced state vector. Due to the small space projection, this inversion is not a computationally expensive operation.

The reduced state vector obtained from (7) is then used in (8) to compute the current flowing through the voltage source. The imaginary part of the current gives the inductance of the spiral, $L_{\text {ind }}$ and the ratio of imaginary and real part yields quality factor $Q$ as in equations (9) - (11).

$$
\begin{gathered}
Z_{\text {ind }}=V_{\text {src }} / y \\
L_{\text {ind }}=\frac{\operatorname{Im}\left(Z_{\text {ind }}\right)}{\omega} \\
Q=\frac{\omega L_{\text {ind }}}{R_{\text {ind }}}=\frac{\operatorname{Im}\left(Z_{\text {ind }}\right)}{\operatorname{Re}\left(Z_{\text {ind }}\right)}
\end{gathered}
$$

\section{Modeling Substrate in the RF Inductors}

In this section, we describe how the substrate parasitics are computed in our simulator. A Green Function based FFT technique is given in $[8,9]$ for modeling substrate parasitics. In this approach, the substrate resistive problem is solved using an equivalent dielectric problem. In the resistive problem, the parasitics consist of resistors and relate the voltage on a contact and its current. While in the dielectric case, the parasitics are conductors and relate contact potentials to contact charges. The Green Function relates the potential on one contact to the charge induced on the neighboring contacts by equation (12). By considering all combinations on $\mathrm{N}$ contact problem, we obtain a $N \times N$ matrix relating the vector of potentials $(\Phi)$ on various contacts to charge vector Q, by equation (13). Matrix C in (13) is known as the coefficient of induction matrix. Its inverse is called coefficient of potential matrix. From the coefficient of induction matrix, we can compute the capacitance between two contacts using equations (14) and (15). 


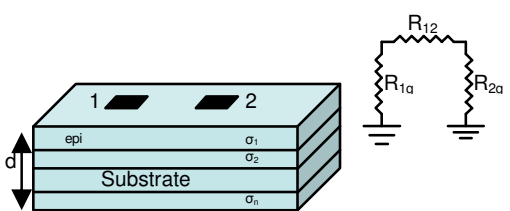

Fig 2: Substrate parasitics

Finally the problem is again transformed into the equivalent resistive problem giving the substrate resistance between two contacts and resistance between the contact and ground using equation (16).

$$
\begin{gathered}
\phi(r)=\int_{V} \rho\left(r^{\prime}\right) G\left(r, r^{\prime}\right) d^{3} r^{\prime} \\
{[Q]=[c][\phi]} \\
C_{i j}=-c_{i j} \\
C_{i g}=c_{i i}+\left(\sum_{j=1}^{N} c_{i j}\right) \\
R_{i j}=\frac{1}{C_{i j}}
\end{gathered}
$$

Figure 2 shows a simple two contact configuration and the result of the result of the system of equations (12) through (16). We use the same technique to extract the resistive mesh underlying the inductor, which is then integrated in the PEEC formulation to yield the complete inductance model. The parameterization and model reduction is then performed on the combined circuit equation to obtain a substrate-aware reduced order equation.

\section{The Algorithm}

In this section we propose a technique to combine a substrate contacts resistive mesh with the PEEC mesh of the inductor.

The algorithm works as follows. First, the inductor is divided into filaments and panels. The filaments having the same coordinates in the direction of flow of current are grouped into sections. The lower surface of each section is projected on the substrate (figure 3 ). This projection determines the substrate contacts that are modeled separately using the Green function based FFT technique. The resistance to ground and inter-contact resistance in absence of current flowing inductor on the top is computed in this way. The resistive mesh obtained from the substrate is finally combined with the mesh obtained from the discretization of the inductor. The complete model is then parameterized and reduced using a technique that will be explained in detail in section 5 .

\section{Discretization}

The conductors forming a spiral should be discretized to properly take into account the skin and edge effects of the current. Due to skin effects, the current flow lines are distorted near the edges. In addition there is charge crowding near the surface. To model these two effects, the discretization near the surface and edge is more dense than the discretization deep inside the surface of conductor. In our simulator, we give the designer complete freedom to choose the pattern of discretization.
Each conductor has to be divided into sections along its length. Each section is sub-divided into filaments of same length as section but width and depth may vary to properly account for skin and edge effects.

The surfaces of the filaments forming the outer surface of the inductor are the panels. The lower surface of each section is mirrored on the substrate to give equivalent substrate contacts (figure 3). The discretization is performed only once per inductor. All the sub-divisions are yielded as needed by the PEEC model.

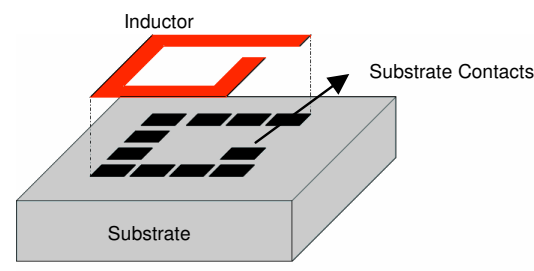

Fig 3: Spiral mapping on the substrate

\section{Substrate Resistive Mesh}

The resistive mesh of the substrate contacts is computed using the technique described in section 3.

Since, each contact corresponds to one section of the inductor, we can represent the combined circuit as shown in figure 4. 6 types of meshes exists:

a) Meshes that only have filaments (with current loop $I_{m}^{f}$ ).

b) Meshes with both panels and filaments $\left(f^{f p}{ }_{m}\right)$.

c) Meshes with filament and contact-to-contact substrate resistance $\left(I_{m}^{f_{s}}\right)$.

d) Meshes with contact-to-contact and contact-to-ground resistances $\left(I^{g g}\right)$.

e) Meshes with only contact-to-contact substrate resistances $\left(I_{m}^{s}\right)$.

f) Meshes that only have panels $\left(I^{p}{ }_{m}\right)$.

We then write the mesh equations for all the meshes to obtain the system of equations (17)-(18). The first six rows of (17) correspond to the meshes listed above in (a)-(f). The $7^{\text {th }}$ row gives the potential conservation across panels in each section. The last row defines the potential drop across the coupling capacitances between the inductors and the substrate.

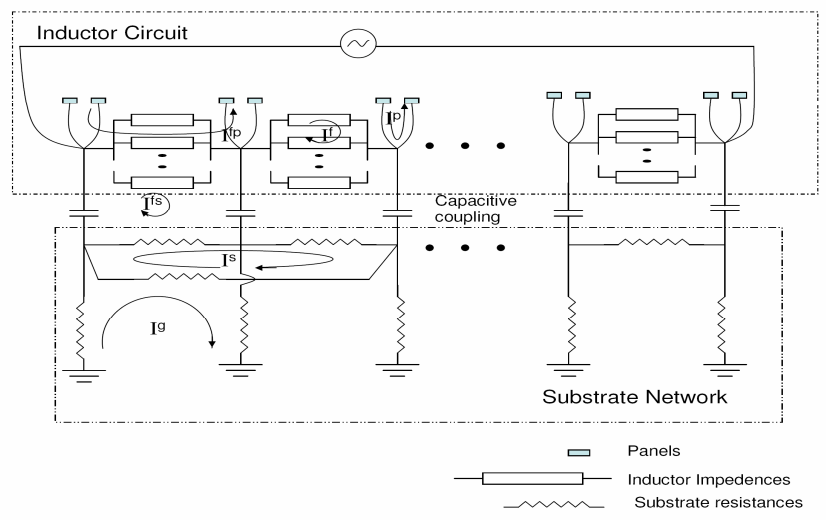

Fig4: Combined substrate parasitic and spiral PEEC network 


$$
\left[\begin{array}{cccccc}
Z_{m}^{f} & Z_{m}^{f p} & Z_{m}^{f p} & 0 & 0 & 0 \\
Z_{m}^{f p^{T}} & Z_{m}^{p} & Z_{m}^{f p} & 0 & 0 & 0 \\
Z_{m}^{f p^{T}} & Z_{m}^{p} & Z_{m}^{f p}+Z_{m}^{f s} & -Z_{m}^{g s} & -Z_{m}^{f s} & 0 \\
0 & 0 & -Z_{m}^{f s^{T}} & Z_{m}^{g} & Z_{m}^{f s} & 0 \\
0 & 0 & -Z_{m}^{f s^{T}} & Z_{m}^{f s^{T}} & Z_{m}^{s} & 0 \\
0 & 0 & 0 & 0 & 0 & 0 \\
0 & -p M p^{p s^{T}} & 0 & 0 & 0 & -p M^{p^{T}} \\
0 & 0 & -p_{c} M^{c^{T}} & 0 & 0 & 0 \\
& & & s L x=-R x+B V_{s r c}
\end{array}\right.
$$$$
\left.\begin{array}{cc}
0 & 0 \\
M^{p s} & 0 \\
0 & M^{c s} \\
0 & 0 \\
0 & 0 \\
M^{p} & 0 \\
s I & 0 \\
0 & s I
\end{array}\right]\left[\begin{array}{c}
I_{m}^{f} \\
I_{m}^{f p} \\
I_{m}^{f s} \\
I_{m}^{s g} \\
I_{m}^{s} \\
I_{m}^{p} \\
V_{b}^{p} \\
V_{b}^{c}
\end{array}\right]=\left[\begin{array}{c}
V_{b}^{f} \\
V_{b}^{f p} \\
V_{b}^{f s} \\
V_{b}^{s g} \\
V_{b}^{s} \\
V_{b}^{p} \\
0 \\
0
\end{array}\right]
$$

In equation (17), $Z$ terms are the impedance in the respective meshes. $p$ 's are the coefficient of potential matrices relating the charge to potential on panels and are computed using equation (19). $p_{c}$ is the coefficient of potential matrix for the coupling capacitance between the inductor and the substrate and is computed using equation (20).

$$
\begin{aligned}
P_{i j} & =\frac{1}{A_{j} 4 \pi \varepsilon_{0}} \int_{P_{j}^{\prime}} \frac{1}{\left\|r_{p_{i}}-r^{\prime}\right\|} d V^{\prime} \\
P_{c}^{i j} & =\left\{\begin{array}{cl}
1 / C_{i} & \text { if } i=j \\
0 & \text { else }
\end{array}\right.
\end{aligned}
$$

The $Z$ terms in the inductor network consist of inductors and resistances, the values of which are computed using equations (21) and (22) respectively.

$$
\begin{gathered}
L_{i j}=\frac{\mu}{4 \pi a_{i} a_{j}} \int_{V_{i}} \int_{V_{j}} \frac{1_{i} \bullet 1_{j}}{\left\|r-r^{\prime}\right\|} d V^{\prime} d V \\
R_{i i}=\frac{l_{i}}{\sigma a_{i}}
\end{gathered}
$$

The $Z$ terms in the substrate networks only have a resistive component, which is computed using the substrate simulator.

By separating out the terms multiplying with $s$ in (17) we obtain the equation (18). In this equation $L$ and $R$ give the imaginary and real part of the impedances of the circuit given in figure 4 .

\section{Parameterization and Model Reduction}

We show now how we can parameterize equation (18). Since $L$ and $R$ matrices in (18) are the function of width of the conductor $(W)$ and the separation between turns $(d)$, we can parameterize these two matrices in terms of $W$ and $d$.

The first step towards parameterization, is selecting the basis functions for expanding the $L$ and $R$ matrices. We will exploit least square estimation, so we select the following as the basis functions:

$$
1, W, d, W^{2}, W d, d^{2}
$$

Expanding $L$ and $R$ we get the following estimator:

$$
\begin{aligned}
& L(W, d) \approx L_{0,0}+W L_{1,0}+d L_{0,1}+W^{2} L_{2,0}+W d L_{1,1}+d^{2} L_{0,2} \\
& R(W, d) \approx R_{0,0}+W R_{1,0}+d R_{0,1}+W^{2} R_{2,0}+W d R_{1,1}+d^{2} R_{0,2}
\end{aligned}
$$

$L_{i, j}$ and $R_{i, j}$ in equations (23) and (24) are computed using the least-square estimation. We choose few sample points, say 8 , in our case and then estimate $L_{i, j}$ 's using equation (25).

$$
\left[\begin{array}{cccccc}
1 & W_{1} & d_{1} & W_{1}^{2} & W_{1} d_{1} & d_{1}^{2} \\
1 & W_{2} & d_{2} & W_{2}^{2} & W_{2} d_{2} & d_{2}^{2} \\
1 & W_{3} & d_{3} & W_{3}^{2} & W_{3} d_{3} & d_{3}^{2} \\
1 & W_{4} & d_{4} & W_{4}^{2} & W_{4} d_{4} & d_{4}^{2} \\
1 & W_{5} & d_{5} & W_{5}^{2} & W_{5} d_{5} & d_{5}^{2} \\
1 & W_{6} & d_{6} & W_{6}^{2} & W_{6} d_{6} & d_{6}^{2} \\
1 & W_{7} & d_{7} & W_{7}^{2} & W_{7} d_{7} & d_{7}^{2} \\
1 & W_{8} & d_{8} & W_{8}^{2} & W_{8} d_{8} & d_{8}^{2}
\end{array}\right]\left[\begin{array}{c}
L_{0,0}^{i, j} \\
L_{1,0}^{i, j} \\
L_{0,1}^{i, j} \\
L_{2,0}^{i, j} \\
L_{1,1}^{i, j} \\
L_{0,2}^{i, j}
\end{array}\right]=\left[\begin{array}{c}
L_{1}^{i, j} \\
L_{2}^{i, j} \\
L_{3}^{i, j} \\
L_{4}^{i, j} \\
L_{5}^{i, j} \\
L_{6}^{i, j} \\
L_{7}^{i, j} \\
L_{8}^{i, j}
\end{array}\right]
$$

Making a Taylor series expansion of equation (18) around an expansion point $s_{0}$, and using the parameters of $L$ and $R$ in equation (23) and (24), we get the following 12 new parameters:

$$
\begin{array}{ll}
s_{1}=W & E_{0}=s_{0} L_{0,0}+R_{0,0} \\
s_{2}=d & E_{1}=s_{0} L_{1,0}+R_{1,0} \\
s_{3}=W^{2} & E_{2}=s_{0} L_{0,1}+R_{0,1} \\
s_{4}=W d & E_{3}=s_{0} L_{2,0}+R_{2,0} \\
s_{5}=d^{2} & E_{4}=s_{0} L_{1,1}+R_{1,1} \\
s_{6}=s & E_{5}=s_{0} L_{0,2}+R_{0,2} \\
s_{7}=s W^{2} & E_{6}=s_{0} L_{0,0} \\
s_{8}=W & E_{7}=s_{0} L_{0,1} \\
s_{9}=s W^{2} & E_{8}=s_{0} L_{1,0} \\
s_{10}=s W^{2} & E_{9}=s_{0} L_{2,0} \\
s_{11}=s d^{2} & E_{10}=s_{0} L_{1,1} \\
& E_{11}=s_{0} L_{2,0}
\end{array}
$$

In the above equations $s(=j \omega)$ is the frequency of operation. Substituting these equations in (18), we obtain the final equation as:

$$
\left(s_{0} E_{0}+s_{1} E_{1}+\ldots \ldots+s_{11} E_{11}\right) x=B V_{s r c}
$$

Applying the model reduction to this equation as described in [2], equation (18) can be re-written as equation (7). The projection matrix $V$ in (7) is computed so as to span the krylov subspace in equation (27). 


$$
F_{k_{2}, \ldots, k_{p}}^{m}\left(M_{1, \ldots, M_{p}}\right)=\left\{\begin{array}{ccc}
0 & \text { if } & k_{i} \notin\{0,1, \ldots ., m\} i=2, \ldots ., p \\
0 & \text { if } & k_{2}+\ldots .+k_{p} \notin\{0,1, \ldots, m\} \\
I & \text { if } & m=0 \\
M_{1} F_{k_{2}, \ldots, k_{p}}^{m-1}\left(M_{1, \ldots, M_{p}}\right)+M_{2} F_{k_{2-1}, \ldots, k_{p}}^{m-1}\left(M_{1, \ldots, M_{p}}\right)+\ldots \ldots .+M_{p} F_{k_{2}, \ldots, k_{p-1}}^{m-1}\left(M_{1, \ldots, M_{p}}\right) & \text { otherwise }
\end{array}\right\}
$$

$$
\begin{gathered}
\operatorname{col} \operatorname{span}(V)=\operatorname{span}\left\{\bigcup_{m=0}^{m_{q}} \bigcup_{k_{2}=0}^{m-\left(k_{p}+\ldots k_{3}\right.} \ldots \bigcup_{k_{p}=0}^{m} F_{k_{2}, \ldots, k_{p}}^{m}\left(M_{1, \ldots, M_{p}}\right) b\right\} \\
b=E_{0}^{-1} B \\
M_{i}=E_{0}^{-1} E_{i}
\end{gathered}
$$

In equation (27), $\mathrm{m}_{\mathrm{q}}$ is the number of moments to be matched and ' $F$ ' is computed using equation (30). With reduced model order equation in (7), we can get the real and imaginary components of current flowing through the inductor for each configuration of variables $s, W$ and $d$. The inductance and quality factor is then computed using the equations (9), (10) and (11).

\section{Results}

The $0.18 \mu \mathrm{m}$ six-metal CMOS technology is used for simulating the RF Inductors. The substrate is 400 um deep with the resistivity of approx $10 \Omega . \mathrm{cm}$. The spiral thickness is $2 \mu \mathrm{m}$, and the oxide thickness between the spiral and the substrate is approximately $7.5 \mu \mathrm{m}$.

We choose the following 6 points $(W, d)$ for interpolation:

$$
\begin{gathered}
(5,5),(5,6),(5,7), \\
(15,5),(15,6),(15,7)
\end{gathered}
$$

In figure 6 are plotted the inductances for 2.5 turns spiral with thickness of $14.5 \mu \mathrm{m}$ and separation varying from $2 \mu \mathrm{m}$ to $6 \mu \mathrm{m}$. The bounding box for the spiral is $240 \times 240 \mu \mathrm{m}$ fabricated on $1 \times 1 \times 0.4 \mathrm{~mm}$ substrate. Figure 7 plots the spiral with varying number of turns. The figure 5 compares the simulated data for 2 turns spiral with and without the substrate. Figure 8 plots the measurements in [12] for 2.5 turns, $14.5 \mu \mathrm{m}$ width and $2 \mu \mathrm{m}$ separation spiral in the same technology as used for other results in this paper. The inductance measurements match well with our simulations. The discrepancy between the Q's is due to the lack of model for the via resistance. This has limited impact on inductance while it is important for evaluating Q.

The runtime for computation can be divided into three tasks:

a) Computation of substrate resistances.

b) Computation of Reduced Modeled Equations.

c) Computation of Inductance and Quality Factor from reduced equations.

The tasks (a) and (b) are repeated for every configuration of inductors and chip. Once the reduced equations are computed, different width, separation and frequency of operation can be modeled in task (c). Every iteration of task (c) takes few milliseconds. The task (b) takes the maximum computation time in the simulator and the CPU time for this task goes from 20 (1.5 turns) minutes to 1 hour (2.5 turns). Since, only the task (c) lies in the optimization loop of the design flow, we are not much bothered about the CPU time of task (a) and task (b). Hence, the parameterization leads to significant speedup of design optimization cycle.

\section{Conclusions}

In this paper we have presented the technique for substrate-aware parameterization of spiral inductors. We first provide the discretization technique for inductors, which is favorable for the substrate noise computation. Then, we describe how these substrate parasitics can be included in the PEEC. Finally the parameterization and model reduction techniques are described for the resulting circuit. Simple least-square estimation techniques are used for parameterization, with sample points randomly selected. The suitability of the model is demonstrated through examples and comparisons to measurements. The impact of substrate parasitics is also shown through comparisons. The accuracy of the model can be improved by selecting the sample points based on more scientific approach like Chebyshev's approximation technique. The model proposed in this paper works best for high resistivity substrate, because the eddy current effects are not yet addressed in the modeling technique.

\section{ACKNOWLEDGMENTS}

The project was entirely supported by grant of the Swiss National Science Foundation - Grant Nr.: 620-066110. The authors would also like to thank Luca Daniel for useful discussions on model reduction algorithms.
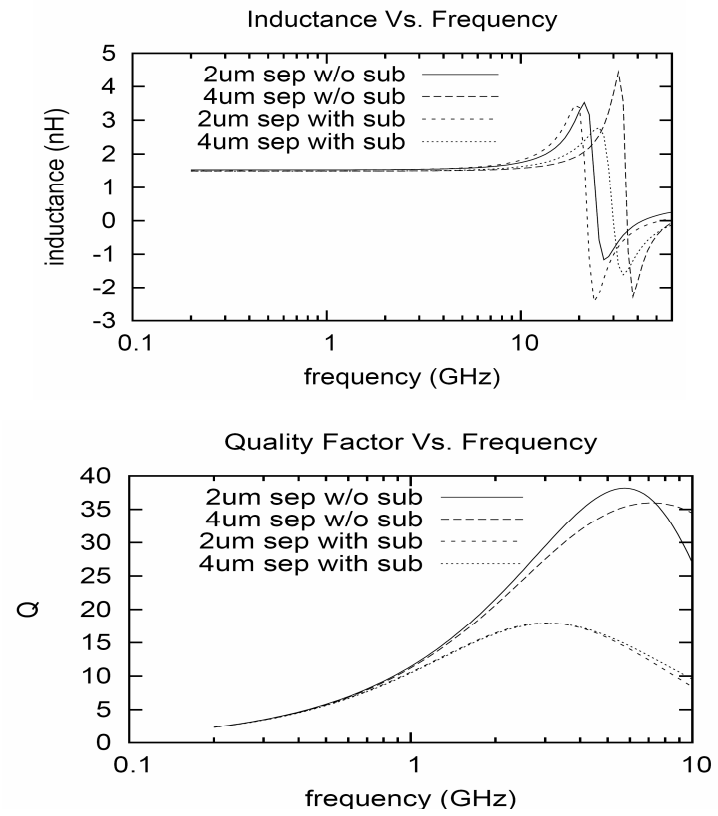

Figure 5: Simulated Inductance and Quality Factor for 2 turns, $14.5 \mu \mathrm{m}$ width, with and without substrate noise 

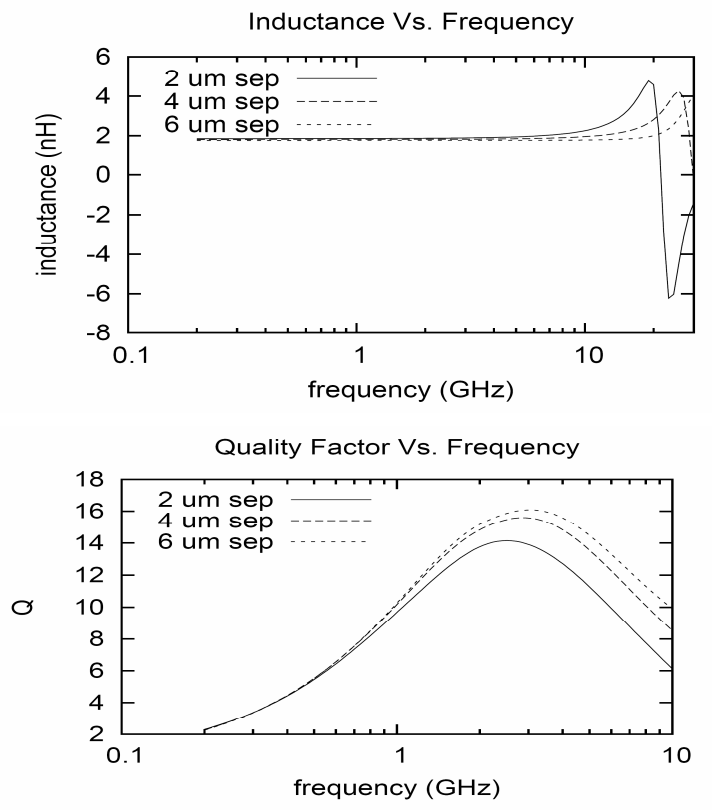

Figure 6: Simulated Inductance and Quality Factor for 2.5 turns, $14.5 \mu \mathrm{m}$ width and $2 \mu \mathrm{m}$ separation
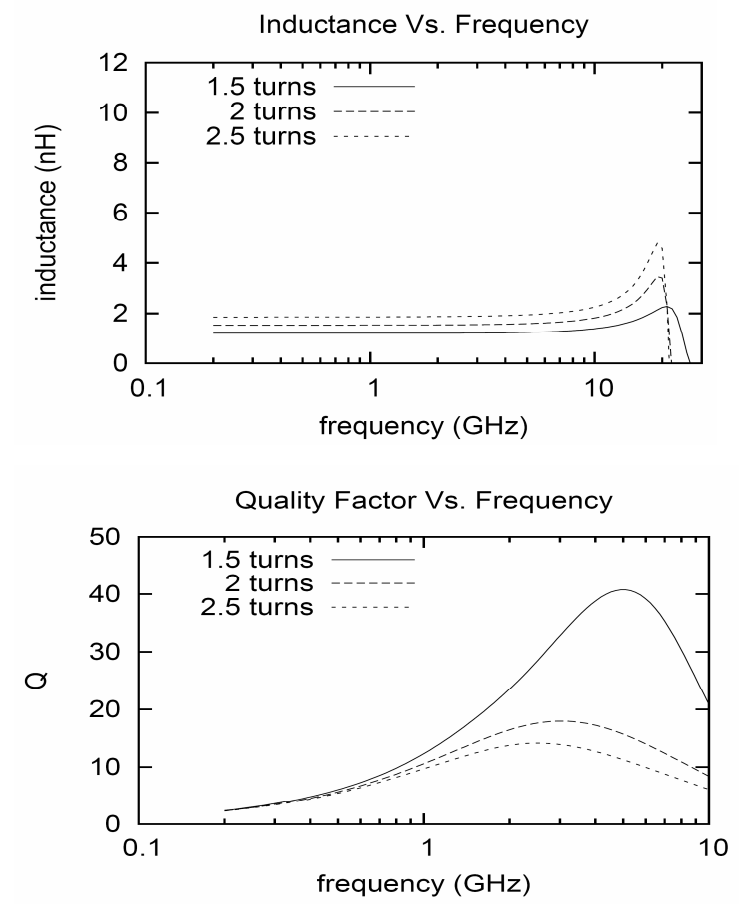

Figure 7: Inductance and Quality Factor for $14.5 \mu \mathrm{m}$ width and $2 \mu \mathrm{m}$ seperation width,

\section{REFERENCES}

[1] L. Daniel and J. K. White, "Automatic Generation of Geometrically Parameterized Reduced Order Models for Integrated Spiral RFInductors", Proc. IEEE BMAS, October 2003.
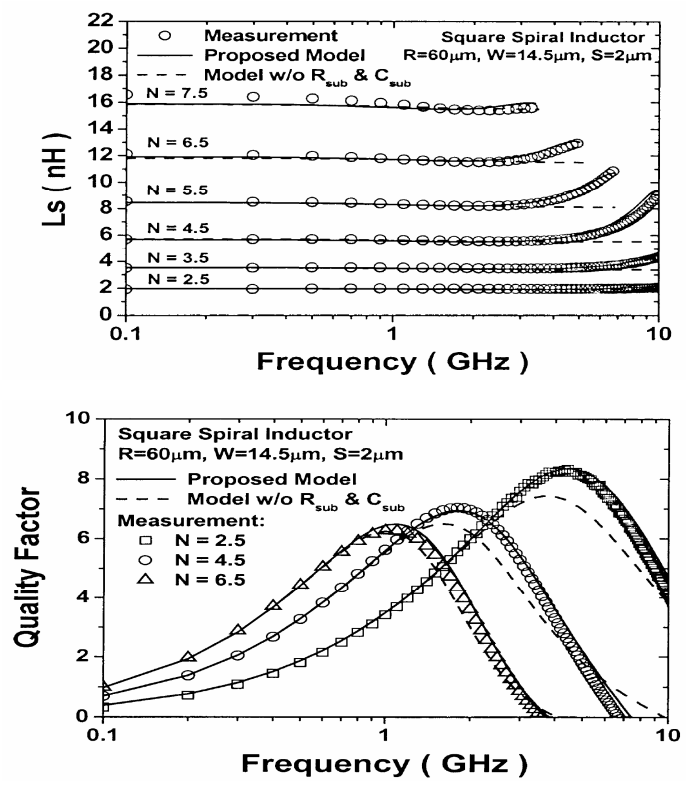

Figure 8: Measured Inductance and Quality for $14.5 \mu \mathrm{m}$ width and $2 \mu \mathrm{m}$ sep. Courtesy [12]

[2] L. Daniel et al., "A Multiparameter Moment-Matching ModelReduction Approach for Generating Geometrically Parameterized Interconnect Performance Models", Trans. on CAD of Integrated Circuits and Systems, Vol. CAD-23, pp. 678-693, May 2004.

[3] Y. Liu, L. T. Pileggi, and A. Strojwas, "Model Order-Reduction of RCL Interconnect Including Variational Analysis", Proc. ACM/IEEE DAC, pp. 201-206, June 1999.

[4] M. Kamon. "Fast Parasitic Extraction and Simulation of Threedimensional Interconnect via Quasistatic Analysis", PhD thesis, Massachusetts Institute of Technology, Cambridge, MA, January 1998.

[5] A. E. Ruehli. Equivalent circuit models for three dimensional multiconductor systems. IEEE Trans. on Microwave Theory and Techniques, vol. 22, pp. 216-221, March 1974.

[6] M. Kamon, N. Marques, and J. K. White. FastPep: a fast parasitic extraction program for complex three-dimensional geometries. In Proc. of the IEEE/ACM ICCAD,San Jose, CA, November 1997.

[7] M. Kamon, M. J. Tsuk, and J. K. White. FASTHENRY: A multipole-accelerated 3-D inductance extraction program. IEEE Trans. on Microwave Theory and Techniques, vol 42, pp. 1750-8, September 1994.

[8] R. Gharpurey and R.G. Meyer, "Modeling and Analysis of Substrate Coupling in Integrated Circuits", JSSC, Vol. 31, No. 3, pp. 344-353, March 1996.

[9] A. M. Niknejad and R. G. Meyer, "Analysis, design, and optimization of spiral inductors and transformers for Si RF IC's," IEEE J. Solid-State Circuits, vol. 33, pp. 1470-1481, Oct. 1998.

[10] M. Chou and J.K. White, "Efficient Formulation and Model-Order Reduction for the Transient Simulation of Three-dimensional VLSI Interconnect," IEEE Trans. on Computer-Aided Design, December 1997, vol.16, no. 12, pp. 1454-1476.

[11] Y. Massoud and J. White, "Simulation and Modeling of the Effect of Substrate Conductivity on Coupling Inductance and Circuit Crosstalk," IEEE Transactions on Very Large Scale Integration (VLSI) Systems, Vol. 1, No. 3, pp. 286-291, June 2002.

[12] J. Gil and H. Shin, "A Simple Wide-Band On-Chip Inductor Model for Silicon-Based RF ICs". IEEE Trans. on Microwave Theory and Techniques, Vol. 51, No. 9, September 2003.

[13] U. Choudhury et al., "An analytical-model generator for interconnect capacitances," in Proc. IEEE CICC, 1991. 